\title{
Childhood Trauma Exposure Disrupts the Automatic Regulation of Emotional Processing
}

\author{
Hilary A Marusak ${ }^{1,2}$, Kayla R Martin ${ }^{3}$, Amit Etkin ${ }^{4,5}$ and Moriah E Thomason*,2,6 \\ 'Department of Psychiatry and Behavioral Neurosciences, Wayne State University School of Medicine, Detroit, MI, USA; ${ }^{2}$ Merrill Palmer Skillman \\ Institute for Child and Family Development, Wayne State University, Detroit, MI, USA; ' Wayne State University School of Medicine, Detroit, MI, \\ USA; ${ }^{4}$ Department of Psychiatry and Behavioral Sciences, Stanford University School of Medicine, Stanford, CA, USA; ${ }^{5}$ Sierra-Pacific Mental IIIness \\ Research, Education, and Clinical Center (MIRECC) Veterans Affairs Palo Alto Health Care System, Palo Alto, CA, USA; ${ }^{6}$ Department of \\ Pediatrics, Wayne State University School of Medicine, Detroit, MI, USA
}

Early-life trauma is one of the strongest risk factors for later emotional psychopathology. Although research in adults highlights that childhood trauma predicts deficits in emotion regulation that persist decades later, it is unknown whether neural and behavioral changes that may precipitate illness are evident during formative, developmental years. This study examined whether automatic regulation of emotional conflict is perturbed in a high-risk urban sample of trauma-exposed children and adolescents. A total of 14 trauma-exposed and 16 age-, sex-, and IQ-matched comparison youth underwent functional MRI while performing an emotional conflict task that involved categorizing facial affect while ignoring an overlying emotion word. Engagement of the conflict regulation system was evaluated at neural and behavioral levels. Results showed that trauma-exposed youth failed to dampen dorsolateral prefrontal cortex activity and engage amygdala-pregenual cingulate inhibitory circuitry during the regulation of emotional conflict, and were less able to regulate emotional conflict. In addition, trauma-exposed youth showed greater conflict-related amygdala reactivity that was associated with diminished levels of trait reward sensitivity. These data point to a trauma-related deficit in automatic regulation of emotional processing, and increase in sensitivity to emotional conflict in neural systems implicated in threat detection. Aberrant amygdala response to emotional conflict was related to diminished reward sensitivity that is emerging as a critical stress-susceptibility trait that may contribute to the emergence of mental illness during adolescence. These results suggest that deficits in conflict regulation for emotional material may underlie heightened risk for psychopathology in individuals that endure early-life trauma.

Neuropsychopharmacology (2015) 40, 1250-1258; doi:I0.1038/npp.20I4.3I I; published online 7 January 2015

\section{INTRODUCTION}

Converging evidence suggests that trauma exposure, particularly in early life, fundamentally alters the way emotional information is processed and prioritized. Indeed, behavioral studies in children with histories of trauma show enhanced attention to and difficulty in disengaging from emotional stimuli (Tottenham et al, 2010). Early-life trauma exposure is a potent risk factor for neuropsychiatric disorders including anxiety, depression, and posttraumatic stress disorder (Gilbert et al, 2009; Kaufman et al, 2000) that are also hallmarked by abnormalities in the processing and regulation of emotion (Etkin and Wager, 2007). Examining emotion regulation in trauma-exposed children and adolescents (youth) who are at elevated risk for developing emotional psychopathology may illuminate biological

*Correspondence: Dr ME Thomason, Merrill Palmer Skillman Institute for Child and Family Development, Wayne State University, 7I East Ferry Street, Detroit, MI 48009, USA, Tel: +1 313664 2517, Fax: + I 313664 2555, E-mail: moriah@wayne.edu

Received 4 June 2014; revised 12 November 2014; accepted 17 November 2014; accepted article preview online 2I November 2014 pathways that link trauma exposure in early life to subsequent emergence of clinical disorder.

Behavioral studies in trauma-exposed individuals report deficits in cognitive control and emotion regulation (Pechtel and Pizzagalli, 2011; Tottenham et al, 2010), processes that are known to develop across the first two decades of life (Casey et al, 1997). Brain regions subserving these functions (eg, pregenual cingulate cortex (pgACC) and dorsolateral prefrontal cortex (DLPFC)) also show protracted development, and are particularly vulnerable to the deleterious effects of early-life stress (Pechtel and Pizzagalli, 2011). It is therefore critical to identify the neural and behavioral correlates of trauma exposure during a time when brain circuits are still forming, and when interventions can be maximally impactful. Yet, neurobiological research examining emotion regulatory pathways in youth exposed to trauma is limited.

Emerging research supports the notion that behavioral alterations observed in trauma-exposed youth may relate to changes in underlying neurobiological processes. For instance, children with early-life trauma exposure show exaggerated amygdala response to threatening cues (McCrory et al, 2011), and altered connectivity in brain systems relevant for 
detecting and processing emotional information (Gee et al, 2013). Together, it appears that early-life adversity is associated with reorganization of neural circuits in ways that enhance processing of salient emotional stimuli. Altered processing of emotional information may underlie or potentiate emotion regulation deficits reported in individuals with histories of early-life trauma (see Pollak, 2008 for a review).

One way to examine emotion regulatory control processes is through neuropsychological tasks where emotional distracters are in direct conflict with task-relevant emotional information (see Figure 1). Emotionally incongruent stimuli, such as the word 'FEAR' superimposed on a happy face, cause reduced accuracy and greater reaction time (RT) interference (ie, slowdowns). The amount of emotional 'conflict', or interference generated by incongruent stimuli (incongruent minus congruent trials (I-C)), is associated with activity in the amygdala (Etkin et al, 2006), a brain region that monitors and detects salient environmental stimuli (Whalen, 1998), and DLPFC, which may reflect effortful attentional control (Derrfuss et al, 2005).

When individuals engage emotion regulatory systems, they become more prepared to inhibit immediate subsequent conflict. As a result, accuracy is improved and RT interference is reduced for incongruent trials if they are preceded by another incongruent trial rather than a congruent trial (iI-cI; Botvinick et al, 1999; Botvinick et al, 2001). This effect, termed 'conflict regulation', seems to occur outside of conscious awareness and thus is a type of automatic emotion regulation (Etkin et al, 2010). Prior functional MRI (fMRI) and lesion studies show that automatic emotional conflict regulation relies on inhibited processing of emotional distracters through top-down (ie, pgACC) modulatory control of amygdala responsivity (Egner et al, 2008; Etkin et al, 2006; Maier and di Pellegrino, 2012).
Perturbations in this critical emotion regulatory pathway have been observed in adults with anxiety and depression (Etkin and Schatzberg, 2011), and may underlie emotion regulatory deficits observed in adults with early-life trauma (Ford, 2005).

This study examines the spontaneous regulation of emotional processing in a sample of trauma-exposed, urban, low-income, minority youth at high risk for psychopathology. Here, we define childhood trauma as the experience of event(s) that threaten a child's safety (ie, witness violence), undermine their security (ie, neglect), or fragment attachment bonds (ie, abuse). We utilized an adapted version of the emotional conflict task (Etkin et al, 2006) for children, by using salient peer emotion faces. Based on behavioral research in trauma-exposed youth (Tottenham et al, 2010) and prior applications of the emotional conflict task in adults with anxiety and major depressive disorder (Etkin et al, 2010; Etkin and Schatzberg, 2011), we hypothesized that trauma-exposed youth would exhibit greater deficits in ability to ignore emotional distracters that are task irrelevant, and reduced ability to regulate emotional conflict. Our analyses focused on amygdala-pgACC circuitry, given prior work emphasizing that this pathway underlies emotional conflict regulation through inhibition of amygdala reactivity (Etkin et al, 2006). Specifically, we predicted higher amygdala response to conflict, lower pgACC response during conflict regulation, and lower negative amygdalapgACC functional connectivity during conflict regulation in trauma-exposed youth. We also tested for the presence of compensatory regional responses in the DLPFC during conflict regulation-previously observed in depressed but not anxious adults (Etkin and Schatzberg, 2011). Finally, we tested whether neural function mediates the association between trauma exposure and emotional well-being. In particular, we evaluated anxiety and depression symptoms,

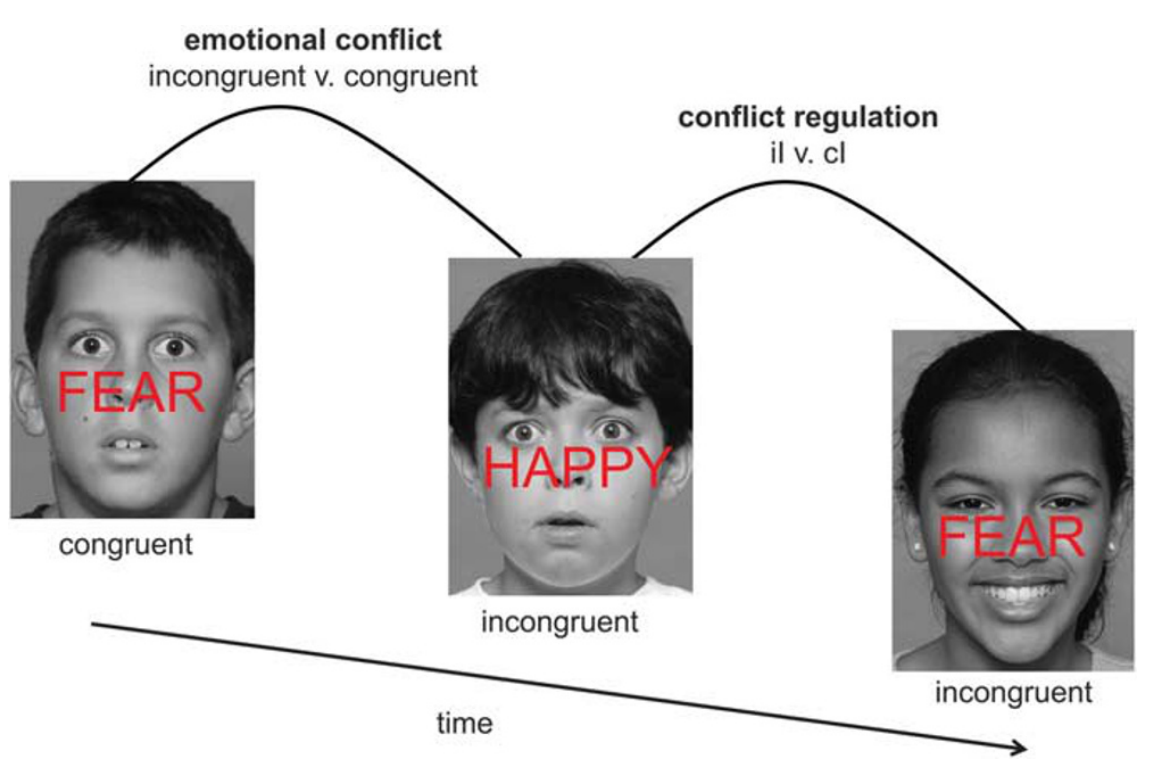

Figure I Emotional conflict task. Participants were instructed to identify the underlying facial emotion (fearful or happy) while ignoring an overlying emotion word ('FEAR' or 'HAPPY'). Trials varied such that emotional distracter words either matched ('congruent' (C)) or conflicted ('incongruent' (I)) with the underlying facial expression. Conflict interference was assessed by contrasting incongruent trials with congruent trials. Conflict regulation was isolated by contrasting postincongruent incongruent (il) with postcongruent incongruent (cl) trials. The task was adapted for children by utilizing an established set of child emotion-face stimuli of varied ethnicities, ages I0-17 years (Egger et al, 20 I I). Importantly, the stimuli used matched the demographics of our study sample and minimized complex relations inherent in adult face stimuli (Marusak et al, 20।3). 
and trait reward sensitivity (RS). The latter was of interest because of emerging research showing that diminished RS is a promising marker of vulnerability to affective disorders following stress (Bogdan et al, 2013). Reduced emotion regulatory ability may be associated with changes in reward function. We therefore tested whether higher amygdalar responses to conflict mediated the association between trauma exposure and low RS.

\section{MATERIALS AND METHODS}

\section{Participants}

A total of 51 children and adolescents, recruited through online advertisements or child psychiatry clinics (Detroit, MI), participated in this fMRI study. Refer to the Supplementary Material for further demographic information and rationale for the study sample. Exclusionary criteria included: English as a second language, lower than a second-grade reading level, history of brain injury, neurological or movement disorders, or presence of MRI contraindications. Parental informed written consent and child/adolescent assent were obtained before participation.

\section{Trauma and Clinical Measures}

Using both parent and child report, participants who endorsed (lifetime) at least one trauma itemized on the Children's Trauma Assessment Center Screen Checklist (source: Michigan Trauma Assessment Center) were categorized as 'trauma'. Number and type of endorsed traumas are provided in Table 1. Participants with movement of $>4 \mathrm{~mm}$ in the scanner ( $n=13 ; 3$ trauma and 10 comparison), accuracy of $<50 \%$ ( $n=5 ; 1$ trauma and 4 comparison), or errors in behavioral data collection ( $n=2 ; 1$ trauma and 1 comparison) were excluded from analyses. Therefore, all neuroimaging data are reported for 14 trauma-exposed and 16 age-, sex-, and IQ-matched comparison youth. For completeness, all participants tested with usable behavioral data were included in behavioral analyses. This resulted in the addition of two participants (1 trauma and 1 comparison) who did not qualify for neuroimaging analyses $(n=15$ trauma; $n=17$ comparison). IQ was assessed using the Kaufman Brief Intelligence Test (KBIT v.2; Kaufman and Kaufman, 2004). Participant ages (9-16 years) were selected to align with the emergence of puberty; puberty has been identified as a time when neuropsychiatric disorders frequently manifest (Angold et al, 1998). Pubertal development was assessed using Tanner staging. Following prior work (Forbes et al, 2009), participants were categorized as pre/early (Tanner stages 1-2) or mid/late pubertal (stages 3-5). Effects of pubertal maturation on trauma-related group differences were examined.

Three validated self-report measures of symptoms and affective traits were administered: (1) the 41-item Screen for Child Anxiety-Related Emotional Disorders (SCR; Birmaher et al, 1997), (2) the 10-item Children's Depression Inventory (CDI; Kovacs, 1985), and (3) the Behavioral Inhibition and Activation Scales (BIS/BAS; Carver and White, 1994). Following prior work (Garner et al, 2012), RS was conceptualized as the BAS component of the BIS/BAS. See Supplementary Material for further information regarding
Table I Participant Demographics by Group

\begin{tabular}{|c|c|c|}
\hline & $\begin{array}{l}\text { Trauma } \\
(n=14)\end{array}$ & $\begin{array}{c}\text { Comparison } \\
(n=16)\end{array}$ \\
\hline Age, mean $(S D)$ & $12.7(2.09)$ & $12.76(2.21)$ \\
\hline Sex (female), $n(\%)$ & $10(71.4)$ & $14(87.5)$ \\
\hline $\mathrm{IQ}$, mean $(\mathrm{SD})$ & $100(13.27)$ & $102.57(13.8)$ \\
\hline \multicolumn{3}{|l|}{ Pubertal maturation, n (\%) } \\
\hline Pre/early pubertal (Tanner stages I-2) & $5(35.7)$ & $5(31.2)$ \\
\hline Mid/late pubertal (Tanner stages 3-5) & $9(64.3)$ & | | (68.8) \\
\hline \multicolumn{3}{|l|}{ Race/ethnicity, n (\%) } \\
\hline African American & $5(35.7 \mathrm{l})$ & $9(56.25)$ \\
\hline Caucasian & $3(21.43)$ & $5(31.25)$ \\
\hline Hispanic & $2(14.29)$ & 0 \\
\hline Biracial & I (7.14) & | (6.25) \\
\hline Not reported & $3(21.43)$ & I (6.25) \\
\hline \multicolumn{3}{|l|}{ Household annual income, n (\%) } \\
\hline$<\$ 40000$ & I। (78.57) & $7(43.75)$ \\
\hline$\$ 40-60000$ & I (7.|4) & $5(31.25)$ \\
\hline$\$ 60-80000$ & | (7.14) & $2(12.5)$ \\
\hline$>\$ 80000$ & 0 & $2(12.5)$ \\
\hline Not reported & । (7.14) & 0 \\
\hline \multicolumn{3}{|l|}{ Type of trauma endorsed, $\mathrm{n}$ (\%) } \\
\hline Physical abuse & $2(14)$ & 0 \\
\hline Neglectful home environment & $3(2 \mid)$ & 0 \\
\hline Exposure to domestic violence & $7(50)$ & 0 \\
\hline Exposure to any other violence not already identified & $7(50)$ & 0 \\
\hline Multiple separations from parent or caregiver & $2(14)$ & 0 \\
\hline Sexual abuse or exposure & $3(21)$ & 0 \\
\hline Anxiety Symptomology (SCR), mean (SD) & $19.29(13.67)$ & $15.18(11.38)$ \\
\hline Depressive Symptomology (CDI), mean (SD) & $2(2.57)$ & $2.12(2.47)$ \\
\hline $\begin{array}{l}\text { Reward Sensitivity (normalized; BAS), } \\
\text { mean (SD) }\end{array}$ & $0.06(0.56)$ & $-0.25(.84)$ \\
\hline Reward responsivity & $17.47(1.34)^{\mathrm{a}}$ & $19.73(1.58)^{\mathrm{a}}$ \\
\hline Fun seeking & $12.21(1.89)$ & $12.53(2.85)$ \\
\hline Drive & $10.85(2.54)$ & $11.33(2.06)$ \\
\hline \multicolumn{3}{|l|}{ Motion during scan ${ }^{\mathrm{b}}$, mean (SD) } \\
\hline Translational mean movement & $0.07(0.06)$ & $0.04(0.02)$ \\
\hline Rotational mean movement & $5.7(5.7)$ & $2.86(2.86)$ \\
\hline Translational RMS & $0.04(0.02)$ & $0.04(0.02)$ \\
\hline Rotational RMS & $0.05(0.03)$ & $0.04(0.03)$ \\
\hline
\end{tabular}

Abbreviations: IQ, intelligence quotient; SCR, screen for child anxiety-related emotional disorders; CDI, children's depression inventory; BAS, behavioral activation subscale of the BIS/BAS scales; RMS, root-mean-square head movement

andicates group comparison is significant at $p \leq 0.05$. The $\chi^{2}$ tests were used for sex, race/ethnicity, puberty, income, and trauma-type comparisons; two-sample t-tests for age, psychopathology, and motion comparisons.

${ }^{\mathrm{b}}$ Translational $(x, y, z)$ movement is reported in $\mathrm{mm}$ and rotational in degrees.

the RS measure. A visual analog scale (VAS) was used to obtain an average rating of fear/anxiety during the MRI visit (repeat measures at 30 -min intervals) as previously described (Thomason et al, 2013).

\section{Experimental Paradigm}

See Figure 1 for task overview, and Supplementary Material for further details. 


\section{Behavioral Analysis}

Accuracy and RT (correct trials) were analyzed in IBM SPSS v.22. Behavioral effects were considered significant at a $p \leq 0.05$ (two-tailed) threshold.

\section{Imaging Data Acquisition}

Functional images were acquired using a 3-Tesla Siemens Verio scanner equipped with a 12-channel head coil (MRI Research Center, Wayne State University). Twenty-nine axial slices were acquired across the whole brain using $\mathrm{T} 2{ }^{*}$ weighted echo-planar imaging (TR: $2000 \mathrm{~ms}$, TE: $25 \mathrm{~ms}$, matrix: $220 \times 220$, flip angle: $90^{\circ}$, voxel size: $3.44 \times 3.44 \times 4$ $\mathrm{mm})$. High-resolution anatomical images were acquired for individuals using a T1-weighted 3D magnetization prepared rapid acquisition gradient echo (MP-RAGE) sequence (TR: $1680 \mathrm{~ms}$, TE: $3.51 \mathrm{~ms}$, orientation: axial, matrix: $384 \times 384$, 176 slices, flip angle: $9^{\circ}$, voxel size: $0.7 \times 0.7 \times 1.3 \mathrm{~mm}$ ).

\section{Movement During the Scan}

During acquisition, Siemens MRI motion correction (MoCo) software was used to retrospectively measure six parameters of rigid-body translation and rotation for each time frame and produce a corrected time series using affine transformation. Following exclusion of high movement participants, movement fell within accepted standards (eg, $<1.5 \mathrm{~mm}$ RMS; Fair et al, 2012; see Table 1).

\section{Imaging Data Analysis}

The fMRI data were preprocessed following procedures described in our prior work (Etkin et al, 2006; Marusak et al, 2013). Refer to the Supplementary Material for further details on image processing.

Group-level random-effects two-sample $t$-tests were used to test for group differences in neural activity during (1) emotional conflict (incongruent minus congruent trials (I-C)) and (2) emotional conflict regulation (postincongruent incongruent minus postcongruent incongruent trials (iI-cI)). The contrast iI-cI isolates activity during conflict trials for which behavior differs by virtue only of expectation created by the previous trial type (ie, previous trial is either congruent or incongruent).

A psychophysiologic interaction analysis (Friston et al, 1997) was conducted to test for group differences in amygdala-pgACC coupling during conflict regulation. We used a bilateral amygdala seed, given that group differences in amygdala activation were observed in both hemispheres and because we did not have a priori predictions about laterality. First, the deconvolved time course was extracted from bilateral amygdala using a mask defined by FSL FIRST subcortical segmentation tool (Patenaude et al, 2011). Then, activity within the amygdala was regressed on a voxel-wise basis against the psychological variable of interest (ie, the interaction term), with the physiological (ie, amygdala time course) and psychological (ie, iI $>$ cI contrast) variables serving as regressors of noninterest. Results were submitted to a random-effects group analysis using two-sample $t$-tests.

Neural results are reported for a priori regions of interest using small-volume correction, $p<0.05$, family-wise error-corrected (FWE). Areas examined include: (1) left and right DLPFC $10 \mathrm{~mm}$ spheres $(x=-47, y=21, z=29$; $x=51, y=21, z=32$ ) and (2) pgACC, utilizing the anatomically defined mask described in our prior work (Etkin and Schatzberg, 2011). All coordinates are reported in Montreal Neurological Institute (MNI) convention. For regions showing group differences, signal was extracted from $4 \mathrm{~mm}$ radius spheres (centered on the peak) to plot the effects of trauma and to test for associations with behavioral and clinical measures. Given that prior research has found trauma-related effects in bilateral amygdala (McCrory et al, 2011), left and right amygdala were also evaluated using FSL FIRST masks (Patenaude et al, 2011). Signal change values were submitted to statistical analyses in SPSS and effects were considered significant at a threshold of $p \leq 0.05$ (two tailed). We also report results from whole-brain voxel-wise analyses at a threshold of $p<0.005$, cluster minimum $=10$ voxels. This threshold was derived from suggested standards for whole-brain comparisons (Lieberman and Cunningham, 2009).

\section{Mediation Analysis}

PROCESS software (v.2.11; Hayes, 2013) implemented in SPSS was used to test for the mediating effects of brain function in the association between trauma exposure and symptoms/affective traits. Indirect effects are considered significant when confidence intervals do not overlap zero (Hayes, 2013).

\section{RESULTS}

Trauma and comparison groups were well matched on age, sex, IQ, race, pubertal maturation, annual household income, and movement during the scan (Table 1). One trauma participant was left-handed. Notably, trauma-exposed youth reported lower levels of reward responsiveness $\left(\mathrm{BAS}_{\mathrm{rr}}\right)$, but did not differ on anxiety or depressive symptoms (see Table 1). VAS scores did not differ between groups $(t(28)=$ $0.314, p=0.76$ ), suggesting that effects reported are not likely influenced by group differences in state fear/anxiety.

\section{Behavior}

Overall task performance. Groups did not differ on overall task accuracy $(t(30)=1.24, p=0.22)$ or $\mathrm{RT}(t(22.69)=1.99$, $p=0.06$; Figure $2 \mathrm{a})$, but a trend in group differences was observed such that trauma participants tended to respond faster than the comparison group.

Effects of task congruency. As expected, emotional conflict (I-C) induced a slowdown in $\mathrm{RT}$ and a reduction in accuracy across the sample, $p$ 's $<0.001$ (see Supplementary Table S1). Groups did not differ on conflict interference (I-C; accuracy: $t(30)=0.9, \quad p=0.37 ; \quad \mathrm{RT}: t(30)=1.77$, $p=0.086)$.

Conflict regulation effects. In the comparison group, participants made $\sim 7 \%$ fewer errors and improved RT by $\sim 20 \mathrm{~ms}$ for repeat conflict trials, a pattern that is consistent with adaptive response to conflict and improved 
a
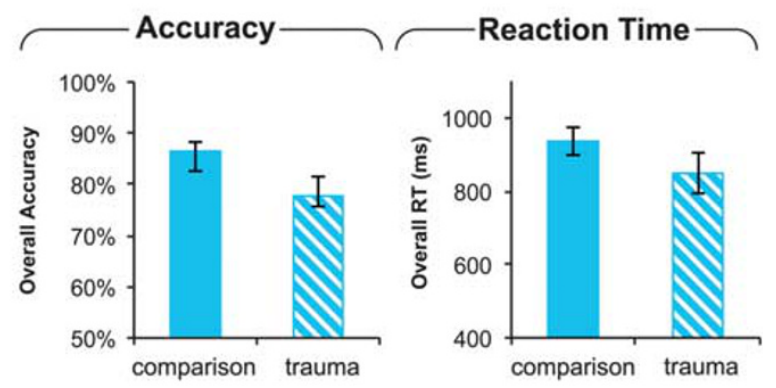

b

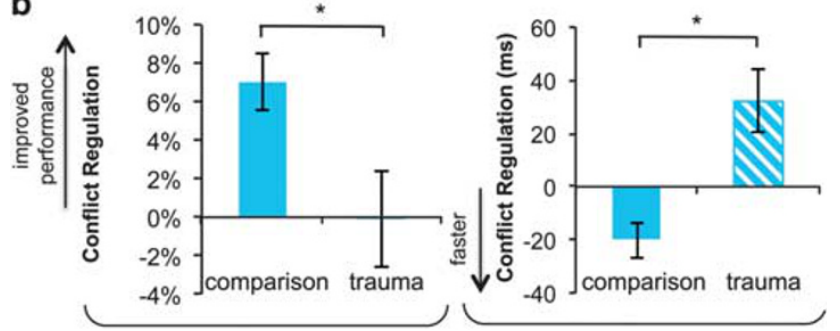

Figure 2 Lower emotion conflict regulatory ability in trauma-exposed youth. (a) No group differences were observed in overall accuracy (left) or reaction time (RT; right). (b) Trauma-exposed youth showed lower ability to regulate emotional conflict. (b, left) Positive values indicate a gain in performance for il relative to $\mathrm{cl}$ trials (il-cl). (b, right) Negative values indicate faster response for il relative to $\mathrm{cl}$ trials (il-cl). $* P \leq 0.05$, twosample t-test. Error bars represent standard error.

performance. In contrast, trauma participants did not show this adaptive gain in performance across repeat interference trials. Trauma participants showed no gain in accuracy; in fact they showed a $\sim 35 \mathrm{~ms}$ reaction time performance decrement when iI trials were compared with cI trials. Furthermore, adaptation differences between groups were significant for both accuracy $(t(30)=2.08, p=0.046)$ and RT $(t(27.25)=2.04, p=0.046$; see Figure $2 \mathrm{~b})$. We discovered no evidence indicating that group differences in conflict regulation were moderated by pubertal maturation (accuracy: $\mathrm{F}(1,28)=0.24, p=0.63$; $\mathrm{RT}: \mathrm{F}(1,28)=0.33, p=0.57)$.

\section{Aberrant Conflict-Related Amygdala Activity in Trauma-Exposed Youth}

Amygdala a priori region of interest analysis revealed greater conflict-related activity $(\mathrm{I}-\mathrm{C})$ in right $(t(28)=2.11$, $p=0.04$, Cohen's $d=0.79)$ and left $(t(28)=2.23, p=0.03$, Cohen's $d=0.84$ ) amygdala in trauma-exposed relative to comparison participants (Figure $3 \mathrm{a}-\mathrm{c}$ ). There were no trauma-by-puberty interactions for left $(\mathrm{F}(1,26)=0.22$, $p=0.65)$ or right $(\mathrm{F}(1,26)=1.83, p=0.19)$ amygdala responses to conflict. No group differences in amygdala activity were observed during conflict regulation (iI-cI).

\section{Trauma-Exposed Youth Show Abnormal Regulation of the DLPFC}

We observed significant small volume-corrected group differences in the left DLPFC during emotional conflict regulation $(x=-46, y=30, z=38, p \mathrm{FWE}=0.027, Z=3.10)$. Relative to comparison youth, trauma participants displayed
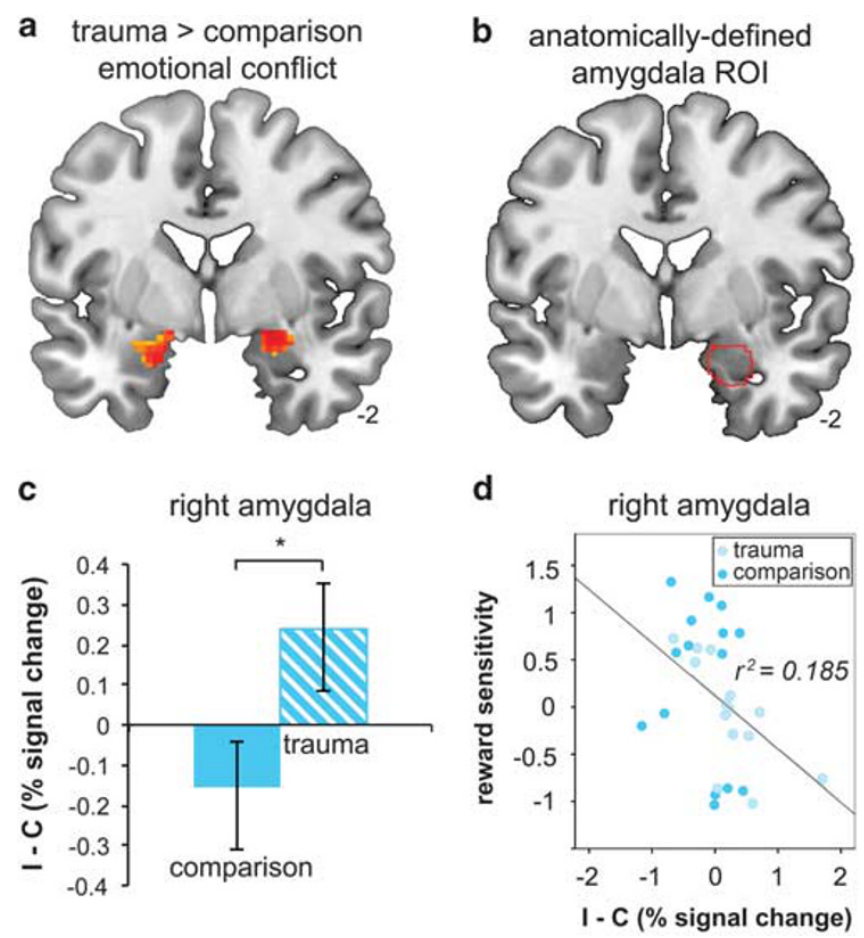

Figure 3 Greater amygdala response to emotional conflict in traumaexposed youth. Greater conflict-related (I-C) bilateral amygdala activity was observed in trauma relative to comparison participants. Results are displayed for the whole brain at $p<0.05$ uncorrected (a). The anatomically defined amygdala region from which average signal was extracted (b) to provide group differences shown in (c). ${ }^{*} P \leq 0.05$ two-sample t-test. Higher conflict-related amygdala reactivity across the sample mediated the association between trauma exposure and diminished reward sensitivity (d). Error bars represent SEM.

elevated left DLPFC activity (Figure 4a). Failure of trauma participants to dampen DLPFC activity in iI trials paralleled their inability to improve accuracy and RT during these trials (see Figure 2b and c) and this association was significant: among trauma-exposed youth, higher DLFPC activity during emotional conflict regulation correlated with reduced ability to improve accuracy during iI relative to $\mathrm{cI}$ trials $(r(14)=-0.585, p=0.028)$. Considerate of possible pubertal effects, we evaluated the trauma-by-puberty interaction for left DLPFC response during conflict regulation; the result was nonsignificant $(\mathrm{F}(1,26)=1.4, p=0.24)$. No differences in pgACC activity were observed between groups.

\section{Absent Amygdala-Pregenual Cingulate Connectivity in Trauma-Exposed Youth}

We observed a significant group difference in amygdala connectivity with the pgACC $(x=6, y=28, z=-4$, $p \mathrm{FWE}=0.016, Z=3.64)$ during emotional conflict regulation (iI-cI; see Figure 4b). Extraction of average connectivity strength within this cluster revealed that the group effect resulted from the predicted negative amygdala-pgACC connectivity in comparison participants during conflict regulation, and this was absent in trauma-exposed youth. Moreover, less negative amygdala-pgACC coupling was 
a trauma > comparison emotional conflict regulation
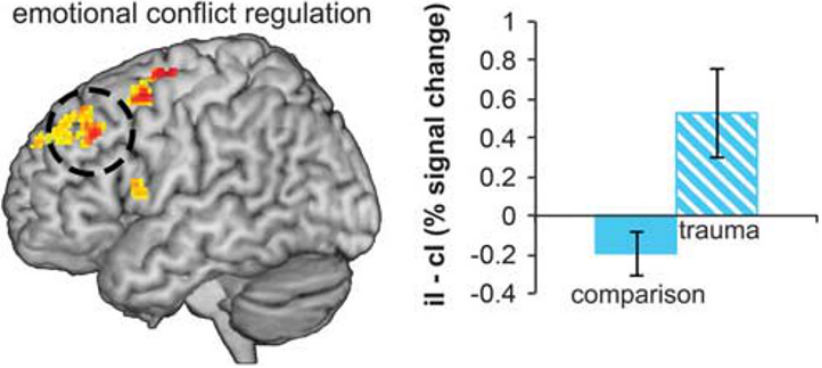

b

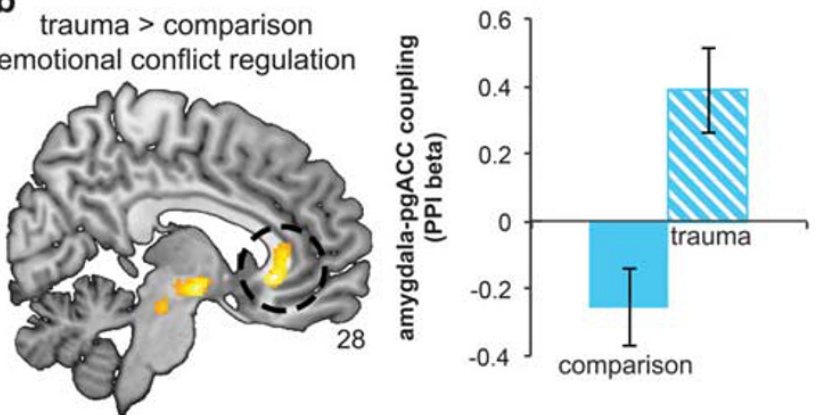

Figure 4 Abnormal regulation of the dorsolateral prefrontal cortex (DLPFC) and disrupted amygdala-pregenual cingulate (pgACC) connectivity in trauma-exposed youth. Increase in DLPFC response is observed in trauma participants (a) and this is related to reduced ability to improve behavioral performance during repeat conflict trials (il-cl; see Figure 2b). Psychophysiologic interaction functional connectivity analysis showed that only comparison participants showed robust negative connectivity between the amygdala and pgACC during emotional conflict regulation (b). Reduced regulatory connectivity was associated with lower ability of trauma participants to improve reaction time during il relative to $\mathrm{Cl}$ trials (il-cl; see Figure 2b). Whole-brain effects displayed at $p<0.01$ uncorrected; results are significant at pFWE $<0.03$.

associated with reduced ability to improve $\mathrm{RT}$ for iI relative to cI trials $(r(30)=0.406, p=0.026)$. The effect of trauma exposure on amygdala-pgACC connectivity was not moderated by pubertal maturation $(\mathrm{F}(1,26)=3.58, p=0.07)$.

\section{Altered Neural Activity Mediates the Relation between Trauma Exposure and Deficits in Reward Sensitivity}

We evaluated whether neural responsivity and trait RS were correlated within brain regions in which neural function differed between groups (ie, amygdala, DLPFC, and pgACC). We observed significant negative correlations between RS and conflict-related activity in the left $(r(29)=-0.38$, $p=0.04)$ and right amygdala $(r(29)=-0.43, p=0.02$; Figure 3d). That is, participants with low levels of RS had higher levels of amygdala response to emotional conflict. These effects were driven by variation in the $\mathrm{BAS}_{\mathrm{rr}}$ subscale (left amygdala: $r(29)=-0.64, p<0.001$; right amygdala: $r(29)=-0.58, p<0.001)$, and held when controlling for anxiety and depression symptoms. RS was not correlated with DLPFC activity or amygdala-pgACC connectivity during emotional conflict regulation ( $p$ 's $>0.1$ ).

We conducted mediation analyses to determine whether conflict-related amygdala reactivity statistically mediated the relationship between trauma exposure and individual variation in trait RS. A mediation relationship (indirect effects) existed for both the left $(\beta=0.08, \mathrm{SE}=0.15$, LLCI (lower limit confidence interval) $=0.001$, ULCI (upper limit confidence interval $)=0.64)$ and right amygdala $(\beta=0.23$, $\mathrm{SE}=0.13, \mathrm{LLCI}=0.02, \mathrm{UCLI}=0.56)$. Direct effects of trauma on RS were not significant, suggesting that amygdala reactivity fully mediated the pathway between trauma exposure and variation in RS. Reversal of this model (trauma exposure $\rightarrow \mathrm{RS} \rightarrow$ amygdala response) yielded nonsignificant indirect effects (right amygdala: $\mathrm{LLCI}=-0.03, \mathrm{ULCI}=0.35$; left amygdala: $\mathrm{LLCI}=-0.03$, ULCI $=0.28$ ), implying that altered neural response mediates RS but not the reverse.

\section{Exploratory Whole-Brain Results}

Results of whole-brain analyses are provided in Supplementary Table S2. Briefly, trauma-exposed youth showed higher activation to conflict (I-C) than comparison youth in regions of the DLPFC, cerebellum, midbrain, primary and secondary visual areas, cuneus, and precentral gyrus. Trauma-exposed youth also showed higher response during conflict regulation (iI-cI) in primary and secondary visual areas, middle frontal gyrus, precentral gyrus, and preceneus. There were no regions with higher activation to conflict or conflict regulation in comparison youth than in trauma-exposed youth at $p<0.005,10$ voxel minimum threshold.

\section{DISCUSSION}

Although research in adults has illuminated lasting neurobiological consequences and deficits in emotion regulation associated with childhood trauma, it is unknown whether neural changes are evident in childhood and adolescence, which may serve as a prelude into emotion problems observed in adulthood. This study tested the hypothesis that childhood trauma exposure alters neural and behavioral systems for detecting and regulating emotional conflict in formative, developmental years. We employed an emotional conflict paradigm in which emotional processing is regulated automatically, and adapted this task for children (see Figure 1). Three main findings emerged. First, compared with their unexposed counterparts, youth with histories of trauma showed greater amygdala reactivity to emotional conflict. Second, trauma-exposed children and adolescents were unable to regulate emotional conflict, indexed by a lack of improvement in accuracy and RT to repeat emotionally incongruent trials. This behavioral deficit was accompanied with a failure to regulate the DLPFC, and an absence of negative regulation-related amygdala-pgACC connectivity in trauma-exposed youth. Third, we found evidence for trait-brain associations: conflict-related amygdala reactivity was associated with diminished levels of RS.

Engagement of the amygdala is thought to confer preferential processing to emotional stimuli (Vuilleumier et al, 2001) so that potential threats can be rapidly detected and evaluated (LeDoux, 1996). Given this role, heightened amygdala reactivity to emotional conflict observed in trauma-exposed youth may reflect alterations in the neural systems that monitor the environment for biologically salient information. There is substantial evidence that 
amygdala reactivity is under inhibitory control of medial prefrontal regions (Ochsner and Gross, 2005), particularly pgACC (Maier and di Pellegrino, 2012). Our results indicate that this mechanism is disrupted in children with early-life trauma, evidenced by (1) reduced ability to regulate emotional conflict, (2) exaggerated amygdala response to conflict, and (3) absence of effective inhibitory control (ie, amygdala-pgACC connectivity). Although we did not observe group differences in pgACC activation during conflict regulation as previously reported in adults with anxiety disorders (Etkin et al, 2010), we did replicate the finding of lower negative functional connectivity between amygdala and pgACC. It is possible that altered pgACC regional responses observed during adulthood result from presence of psychiatric disorder, or compensation resulting from earlier emerging disruptions in amygdala-pgACC circuitry. We recently reported that resting intrinsic connectivity of the amygdala-pgACC regulatory pathway is altered in youth with histories of trauma, suggesting that these deficits are pervasive and are detected even when the individual is not engaging in emotion regulatory processes. Similar deficits in top-down control are observed in adults with high levels of trait anxiety (Hare et al, 2008), generalized anxiety disorder (Etkin et al, 2010), and major depressive disorder (Etkin and Schatzberg, 2011). As trauma-exposed youth in this study were unable to automatically regulate emotional processing, it is possible that children's early experiences may alter attentional thresholds (indexed by amygdala reactivity) in ways that undermine effective emotion regulation (see review by Pollak, 2008). Notably, group differences in amygdala response to conflict occurred in the absence of differences in behavioral interference, suggesting that elevated amygdala reactivity represents a latent neural mechanism that enhances the processing of emotional information.

Prior work in healthy adults emphasizes that the DLPFC is online during conflict detection, and is then dampened during resolution (Etkin et al, 2006). DLPFC activity during conflict resolution in trauma-exposed but not comparison youth suggests aberrant engagement of attentional control. Moreover, persistent activation of the DLPFC in trauma participants correlated with observed behavioral deficits, that is, reduced ability to regulate emotional conflict. It is notable that, similar to trauma-exposed youth in this study, adults with major depression show increased DLPFC activity during emotional conflict regulation, and an absence of amygdala-pgACC inhibitory control in the same task used here (Etkin and Schatzberg, 2011). However, unlike traumaexposed youth, depressed adults were not impaired in their ability to behaviorally regulate emotional conflict. In contrast to our findings, DLPFC activity correlated with better emotion regulatory ability, suggesting that DLPFC recruitment in depressed adults reflects effortful control in support of emotional conflict regulation (Etkin and Schatzberg, 2011). Altogether, our results suggest that dysfunction of the DLPFC observed in trauma-exposed youth may interfere with resolution of emotional conflict and/or represent inefficient neural resources recruited in an effort to overcome this deficit.

A wealth of research shows that early-life adversity is one of the strongest predictors of psychopathology (Gilbert et al, 2009). Although trauma-exposed youth did not show higher levels of anxiety or depression, they reported experiencing decreased positive affect in response to rewarding stimuli (ie, lower levels of $\mathrm{BAS}_{\mathrm{rr}}$ ). This is striking given that diminished RS is emerging as a promising trait marker of disease susceptibility (Bogdan and Pizzagalli, 2006) and severity (Kasch et al, 2002). Deficits in reward processing are particularly relevant for the onset of psychopathology in youth, predicting increases in depressive symptoms (Morgan et al, 2013) and poorer response to evidence-based treatments during adolescence (McMakin et al, 2012). Moreover, changes in reward processing are thought to contribute to key affective and motivational features of anhedonia (Treadway and Zald, 2011).

Our results demonstrate novel associations between earlylife trauma, deficits in emotion regulation, and variation in RS during formative years. Specifically, trauma in early life predicts reduced RS through altered function of the neural systems that process emotion. This is in line with a growing body of literature linking early-life stress to reward processing dysfunction (Bogdan et al, 2013). Lack of positive engagement during childhood and adolescence may contribute to a loss of the normal protection or resilience against traumatic stress. Highlighting the specificity of this effect, no relationships were observed for anxiety or depression, and RS effects held when controlling for these variables.

Study limitations are important to note. First, we tested the effects of trauma in an urban, low-income sample that is predominantly female and African American. One must therefore be cautious when generalizing these effects. Second, sample size was limited, and replication in larger samples is warranted. Third, a high number of participants were excluded because of excess motion and/or low task performance. Although this is a situation common in pediatric studies, future studies examining conflict interference in youth should consider shortening the task length, introducing more breaks, or having a researcher remain in the room with the participant to ensure task compliance and remind them to be still. Fourth, this was a crosssectional study, and thus it is not possible to determine how variation in neural and behavioral responses during conflict regulation affect long-term outcomes. Longitudinal evaluation will be necessary to determine how specific observations herein relate to vulnerability or resilience to trauma in early life.

In summary, we demonstrate trauma-related perturbations in the neural and behavioral systems that underlie emotional conflict regulation in childhood/adolescence. Trauma-exposed youth showed greater amygdala response to emotional conflict, reduced ability to regulate emotional conflict, failure to engage amygdala-pgACC regulatory circuitry, and ineffective DLPFC engagement. These findings imply a simultaneous heightened sensitivity to conflicting emotional information and a lack of regulatory control over emotion processing in youth who have experienced trauma. This 'double hit' is likely to limit the ability of the child to master age-appropriate skills in social and academic domains. We speculate that attentional biases and emotion regulation difficulties may confer elevated risk for psychopathology in youth exposed to trauma. Here, trauma-related changes were detected in systems 
responsible for the automatic regulation of emotion, in line with emerging evidence that the root of emotion regulatory difficulties in psychopathology might be in more spontaneous forms of emotion regulation (Etkin et al, 2010). Our results show trauma-related changes in neural systems that regulate emotional conflict in youth, offering a potential target for future interventions.

\section{FUNDING AND DISCLOSURE}

The authors declare no conflict of interest.

\section{ACKNOWLEDGEMENTS}

We thank Zahid Latif and Yashwanth Katkuri of Wayne State University (WSU) for their assistance in neuroimaging data acquisition, Kayla Martin, Gregory H. Baldwin, Melissa Youmans, Mallory Gardner, Amy Katherine Swartz, Timothy Lozon, Berta Rihan, Stephen Shen, Amy Anderson, and Ali Daher of WSU for assistance in participant recruitment and data collection, Luke Hyde, Arianna Gard, and Megan Martz for feedback on writing, and children and their families who participated in this research. Research reported in this publication was supported, in part, by the Merrill Palmer Skillman Institute and the Department of Pediatrics, Wayne State University School of Medicine, by a NARSAD Young Investigator Award to MET. AE was supported by the Sierra-Pacific MIRECC at the VA Palo Alto Health Care System.

\section{REFERENCES}

Angold A, Costello EJ, Worthman CM (1998). Puberty and depression: the roles of age, pubertal status and pubertal timing. Psychol Med 28: 51-61.

Birmaher B, Khetarpal S, Brent D, Cully M, Balach L, Kaufman J et al (1997). The Screen for Child Anxiety Related Emotional Disorders (SCARED): scale construction and psychometric characteristics. J Am Acad Child Adolesc Psychiatry 36: 545-553.

Bogdan R, Nikolova YS, Pizzagalli DA (2013). Neurogenetics of depression: a focus on reward processing and stress sensitivity. Neurobiol Dis 52: 12-23.

Bogdan R, Pizzagalli DA (2006). Acute stress reduces reward responsiveness: implications for depression. Biol Psychiatry 60: 1147-1154.

Botvinick M, Nystrom LE, Fissell K, Carter CS, Cohen JD (1999). Conflict monitoring versus selection-for-action in anterior cingulate cortex. Nature 402: 179-181.

Botvinick MM, Braver TS, Barch DM, Carter CS, Cohen JD (2001). Conflict monitoring and cognitive control. Psychol Rev 108: 624-652.

Carver CS, White TL (1994). Behavioral inhibition, behavioral activation, and affective responses to impending reward and punishment: the BIS/BAS Scales. J Pers Soc Psychol 67: 319-333.

Casey BJ, Trainor RJ, Orendi JL, Schubert AB, Nystrom LE, Giedd JN et al (1997). A developmental functional MRI study of prefrontal activation during performance of a go-no-go task. J Cogn Neurosci 9: 835-847.

Derrfuss J, Brass M, Neumann J, von Cramon DY (2005). Involvement of the inferior frontal junction in cognitive control: meta-analyses of switching and Stroop studies. Hum Brain Mapp 25: 22-34.

Egger HL, Pine DS, Nelson E, Leibenluft E, Ernst M, Towbin KE et al (2011). The NIMH Child Emotional Faces Picture Set
(NIMH-ChEFS): a new set of children's facial emotion stimuli. Int J Methods Psychiatric Res 20: 145-156.

Egner T, Etkin A, Gale S, Hirsch J (2008). Dissociable neural systems resolve conflict from emotional versus nonemotional distracters. Cereb Cortex 18: 1475-1484.

Etkin A, Egner T, Peraza DM, Kandel ER, Hirsch J (2006). Resolving emotional conflict: a role for the rostral anterior cingulate cortex in modulating activity in the amygdala. Neuron 51: 871-882.

Etkin A, Prater KE, Hoeft F, Menon V, Schatzberg AF (2010). Failure of anterior cingulate activation and connectivity with the amygdala during implicit regulation of emotional processing in generalized anxiety disorder. Am J Psychiatry 167: 545-554.

Etkin A, Schatzberg AF (2011). Common abnormalities and disorder-specific compensation during implicit regulation of emotional processing in generalized anxiety and major depressive disorders. Am J Psychiatry 168: 968-978.

Etkin A, Wager TD (2007). Functional neuroimaging of anxiety: a meta-analysis of emotional processing in PTSD, social anxiety disorder, and specific phobia. Am J Psychiatry 164: 1476-1488.

Fair DA, Nigg JT, Iyer S, Bathula D, Mills KL, Dosenbach NU et al (2012). Distinct neural signatures detected for ADHD subtypes after controlling for micro-movements in resting state functional connectivity MRI data. Front Syst Neurosci 6: 80.

Forbes EE, Hariri AR, Martin SL, Silk JS, Moyles DL, Fisher PM et al (2009). Altered striatal activation predicting real-world positive affect in adolescent major depressive disorder. Am J Psychiatry 166: 64-73.

Ford JDP (2005). Treatment implications of altered affect regulation and information processing following child maltreatment. Psychiatr Ann 35: 410-419.

Friston KJ, Buechel C, Fink GR, Morris J, Rolls E, Dolan RJ (1997). Psychophysiological and modulatory interactions in neuroimaging. NeuroImage 6: 218-229.

Garner KG, Dux PE, Wagner J, Cummins TD, Chambers CD, Bellgrove MA (2012). Attentional asymmetries in a visual orienting task are related to temperament. Cogn Emot 26: 1508-1515.

Gee DG, Gabard-Durnam LJ, Flannery J, Goff B, Humphreys KL, Telzer EH et al (2013). Early developmental emergence of human amygdala-prefrontal connectivity after maternal deprivation. Proc Natl Acad Sci USA 110: 15638-15643.

Gilbert R, Widom CS, Browne K, Fergusson D, Webb E, Janson S (2009). Burden and consequences of child maltreatment in highincome countries. Lancet 373: 68-81.

Hare TA, Tottenham N, Galvan A, Voss HU, Glover GH, Casey BJ (2008). Biological substrates of emotional reactivity and regulation in adolescence during an emotional go-nogo task. Biol Psychiatry 63: 927-934.

Hayes AF (2013). Introduction to Mediation, Moderation, and Conditional Process Analysis: A Regression-Based Approach. Guilford Publications.

Kasch KL, Rottenberg J, Arnow BA, Gotlib IH (2002). Behavioral activation and inhibition systems and the severity and course of depression. J Abnorm Psychol 111: 589-597.

Kaufman AS, Kaufman NL (2004). Kaufman Brief Intelligence TestSecond Edition (KBIT-2). American Guidance Service: Circle Pines, MN.

Kaufman J, Plotsky PM, Nemeroff CB, Charney DS (2000). Effects of early adverse experiences on brain structure and function: clinical implications. Biol Psychiatry 48: 778-790.

Kovacs M (1985). The Children's Depression, Inventory (CDI). Psychopharmacol Bull 21: 995-998.

LeDoux JE (1996). The Emotional Brain: The Mysterious Underpinnings of Emotional Life. Simon \& Schuster.

Lieberman MD, Cunningham WA (2009). Type I and type II error concerns in fMRI research: re-balancing the scale. Soci Cogn Affect Neurosci 4: 423-428. 
Maier ME, di Pellegrino G (2012). Impaired conflict adaptation in an emotional task context following rostral anterior cingulate cortex lesions in humans. J Cogn Neurosci 24: 2070-2079.

Marusak HA, Carre JM, Thomason ME (2013). The stimuli drive the response: an fMRI study of youth processing adult or child emotional face stimuli. NeuroImage 83: 679-689.

McCrory EJ, De Brito SA, Sebastian CL, Mechelli A, Bird G, Kelly PA et al (2011). Heightened neural reactivity to threat in child victims of family violence. Curr Biol 21: R947-R948.

McMakin DL, Olino TM, Porta G, Dietz LJ, Emslie G, Clarke G et al (2012). Anhedonia predicts poorer recovery among youth with selective serotonin reuptake inhibitor treatment-resistant depression. J Am Acad Child Adolesc Psychiatry 51: 404-411.

Morgan JK, Olino TM, McMakin DL, Ryan ND, Forbes EE (2013). Neural response to reward as a predictor of increases in depressive symptoms in adolescence. Neurobiol Dis 52: 66-74.

Ochsner KN, Gross JJ (2005). The cognitive control of emotion. Trends Cogn Sci 9: 242-249.

Patenaude B, Smith SM, Kennedy DN, Jenkinson M (2011). A Bayesian model of shape and appearance for subcortical brain segmentation. NeuroImage 56: 907-922.
Pechtel P, Pizzagalli DA (2011). Effects of early life stress on cognitive and affective function: an integrated review of human literature. Psychopharmacology 214: 55-70.

Pollak SD (2008). Mechanisms linking early experience and the emergence of emotions: illustrations from the study of maltreated children. Curr Dir Psychol Sci 17: 370-375.

Thomason ME, Tocco MA, Quednau KA, Bedway AR, Carre JM (2013). Idle behaviors of the hippocampus reflect endogenous cortisol levels in youth. J Am Acad Child Adolesc Psychiatry 52: 642-652 e641.

Tottenham N, Hare TA, Quinn BT, McCarry TW, Nurse M, Gilhooly $\mathrm{T}$ et al (2010). Prolonged institutional rearing is associated with atypically large amygdala volume and difficulties in emotion regulation. Dev Sci 13: 46-61.

Treadway MT, Zald DH (2011). Reconsidering anhedonia in depression: lessons from translational neuroscience. Neurosci Biobehav Rev 35: 537-555.

Vuilleumier P, Armony JL, Driver J, Dolan RJ (2001). Effects of attention and emotion on face processing in the human brain: an event-related fMRI study. Neuron 30: 829-841.

Whalen P (1998). Fear, vigilance, and ambiguity: initial neuroimaging studies of the human amygdala. Curr Dir Psychol Sci 7: 177-188.

Supplementary Information accompanies the paper on the Neuropsychopharmacology website (http://www.nature.com/npp) 